\title{
TEORIAS NÃO-REPRESENTACIONAIS NA GEOGRAFIA II: MÉTODOS PARA UMA GEOGRAFIA DO QUE ACONTECE
}

DANIEl Paiva $^{1}$

\begin{abstract}
RESUMO - Na sequência de um texto anterior ${ }^{\mathrm{i}}$ sobre os principais contributos teóricos das teorias não-representacionais na Geografia, o objetivo desta síntese bibliográfica é discutir as implicações metodológicas desta nova perspetiva teórica, e os métodos que têm sido reinventados para alcançar os seus propósitos. Nesta síntese, começarei por apresentar o conceito de pensamento em ação que fundamenta as metodologias não-representacionais. De seguida, apresentarei as principais inovações e experimentações ao nível do método. Concluirei com uma breve discussão, na qual se refere as principais contribuições das teorias não-representacionais para a Geografia Cultural contemporânea, bem como as principais críticas que se podem apontar aos estudos existentes neste âmbito.
\end{abstract}

Palavras-chave: Teorias não-representacionais; métodos atmosféricos; métodos móveis; pensamento-em-ação; geografia cultural.

ABSTRACT - NON-REPRESENTATIONAL THEORIES IN GEOGRAPHY II: METHODS FOR A GEOGRAPHY OF WHAT HAPPENS. Following a previous text on the main theoretical contributions of non-representational theories in Geography, the objective of this review article is to discuss the methodological implications of this new theoretical perspective, and the methods that have been reinvented to reach its goals. The article is divided as follows. First, I will present the concept of thought in action, which fundaments non-representational methodologies. Afterwards, I will present the main innovation and experiments method-wise. I will conclude with a discussion on the main contributions of non-representational theories for contemporary Cultural Geography, as well as the main criticism that has been made.

Keywords: Non-representational theories; atmospheric methods; mobile methods; thought-in-action; cultural geography.

Recebido: outubro 2016. Aceite: dezembro 2017.

1 Investigador Associado do Centro de Estudos Geográficos do Instituto de Geografia e Ordenamento do Território da Universidade de Lisboa, R. Branca Edmée Marques, 1600-276, Lisboa, Portugal. E-mail: daniel.paiva@campus.ul.pt 
RÉSUMÉ - LES THÉORIES NON-REPRÉSENTATIONNELLES EN GÉOGRAPHIE II: DES MÉTHODES POUR UNE GÉOGRAPHIE DE «CE QUI SE PASSE». À la suite d'un texte traitant des principales contributions théoriques portant sur ce thème, on discute, dans la présente synthèse bibliographique, les implications méthodologiques de cette nouvelle perspective théorique et les méthodes réinventées pour cela. On présente d'abord le concept de pensée en action, qui est la base des théories non-representationnelles, puis on présente les principales innovations et expériences méthodologiques et on conclu en discutant les principales contributions que les théories non-representationnelles ont apportées à la Géographie culturelle contemporaine, ainsi que les principales critiques qu'on leur a faites.

Mots clés: Théories non-représentationnelles; méthodes atmosphériques; méthodes mobiles; pensée en action; géographie culturelle.

\section{INTRODUÇÃO}

As teorias não-representacionais desestabilizaram o significado de alguns conceitos-chave das ciências sociais, como os conceitos de sujeito, corpo, estrutura ou espaço. Ao invés de os considerar como segmentos bem delimitados do mundo que podem ser estudados individualmente, as teorias não-representacionais vêm sublinhar a sua emergência comum, generativa e relacional nos eventos quotidianos (Thrift, 2008; Anderson \& Harrison, 2010). Embora o aspeto do método nem sempre tenha recebido a mesma atenção que os aspetos relativos à teoria (Vannini, 2015a), vários autores como Thrift (2011), McCormack (2013) ou Vannini (2015b) têm-se dedicado à discussão metodológica no âmbito das teorias não-representacionais.

O meu objetivo neste texto é traçar as principais implicações metodológicas das teorias nãorepresentacionais na geografia, as suas principais inovações, e discutir o seu impacto na Geografia Cultural ${ }^{\mathrm{ii}}$ contemporânea. A síntese divide-se da seguinte maneira. Começarei por apresentar o conceito de pensamento em ação que fundamenta as metodologias não-representacionais. Depois, apresentarei as principais inovações e experimentações ao nível do método, focando-me nos métodos atmosféricos e métodos móveis. Seguir-se-á uma breve discussão, na qual reflito sobre as principais contribuições das teorias não-representacionais para a Geografia Cultural contemporânea, e apresento as principais críticas que se podem apontar aos estudos existentes neste âmbito.

\section{PENSAMENTO EM AÇÃO}

A construção de conhecimento sobre o objeto de estudo nas teorias não-representacionais sustenta-se numa noção de 'pensamento-em-ação' (Anderson \& Harrison, 2010). Isto é, baseia-se na noção de que o conhecimento não é divisível da realidade no qual emerge, contrariando a clássica divisão cartesiana entre representação e realidade, entre mente e corpo. Neste sentido, a produção de conhecimento passa a preocupar-se não apenas com as representações mentais da realidade (e.g. significados, sentimentos, discursos, estruturas), mas também com o modo como essas representações são produzidas corporeamente no decorrer das acções e interacções de que o mundo é constituído (incluíndo práticas, hábitos, afetos). Deste modo, algumas das separações conceptuais acerca do trabalho geográfico esbatem-se. Em primeiro lugar, o foco em aspetos que vão para além da representação também corresponde à consideração do papel de aspetos fisiológicos e neurológicos na ação social, atenuando a divisão entre o biológico e o social (Clough, 2007; Thrift, 2008). Por outro lado, a separação entre corpo e mente 
é diminuída quando se salienta que o pensamento é gerado in locus e in acto, e que é através da presença do corpo no mundo que o sujeito pensa o mundo (Brennan, 2004; Stewart, 2011). Isto leva-nos a um outro limite que é atenuado que é aquele entre sujeito e ambiente. O sujeito como presença no mundo é também um elemento inseparável do mundo, um corpo constantemente atravessado pelos seus elementos como o ar, água, comida, sons, ou tecnologias (Anderson \& Harrison, 2010; Greenhough, 2010; Posteraro, 2014).

Estas considerações obrigam a uma revisão da noção de campo, uma das mais fundamentais na prática da ciência geográfica. Não é nova a noção de que o ponto de vista do investigador é um produto das relações que ele desenvolve, tanto na academia (com colegas de trabalho, em congressos, nas suas leituras) como no campo (com os lugares que visita, com as pessoas com que fala, com a informação que consegue recolher), e como ambas se influenciam mutuamente (Crang \& Cook, 2007). Mas recentemente tem sido também criticada a separação sequencial entre: (i) espaços-tempo de exploração teórica, em que o investigador lê trabalhos de colegas e consulta-os, geralmente na universidade; (ii) espaços-tempo de recolha de dados em que o investigador se desloca a onde ele considera ser o seu campo de análise; e (iii) espaços-tempo de análise de dados, frequentemente de novo na universidade, onde o investigador analisa, reflete e escreve sobre os dados que recolheu no campo. Esta sequenciação do trabalho académico fundamenta-se justamente na divisão cartesiana entre representação e realidade (McCormack, 2013), ou seja, na ideia positivista de que se formam as teorias como representações abstractas, e que é possível depois recolher-se dados reais que nos permitem confirmar ou falsear as teorias. As críticas feministas (e.g. Haraway, 1988) a este modo de produzir conhecimento destacaram o facto de que o conhecimento não emerge do abstracto, mas da perspectiva situada do investigador, e tem sido a partir deste prisma que as teorias não-representacionais têm desenhado as suas metodologias (Anderson \& Harrison, 2010). Logo, a noção de campo é hoje uma noção alargada, que engloba a totalidade dos espaços a partir dos quais o investigador produz conhecimento, sendo assim o campo entendido como "um espaço distribuído e diferenciado composto por relações praticadas entre corpos, textos, tecnologias e materiais. Ao invés de pepitas de informação à espera de serem descobertas, os dados neste campo são agora entendidos como materiais afetivos e co-produzidos" (McCormack, 2013, p. 11)

Neste sentido, o valor das metodologias etnográficas têm sido explorado com um renovado interesse (Vannini, 2015a). Isto está ligado principalmente à possibilidade da observação participante permitir ao investigador explorar a experiência geográfica através do seu próprio corpo. Têm sido valorizadas metodologias que permitem que o próprio investigador seja colocado 'em risco', no centro da ação, e que seja um elemento afetado pela experiência e a prática geográfica (Greenhough, 2010; McCormack, 2013). Este 'risco' não se refere diretamente a uma possível ameaça à integridade física do investigador, mas a um risco epistemológico no qual o investigador deve permitir que a experiência do mundo (re) defina os seus conceitos, refletindo in situ sobre o que acontece de modo a que o conhecimento académico se aproxime do conhecimento prático do mundo para o explicar, sem o reduzir a representações estáticas.

A perspetiva do pensamento em ação implica, como afirmei, uma perspetiva relacional que repense as relações entre o biológico e o social, o corpo e a mente, e o sujeito e ambiente. De seguida, aprofundarei as mudanças no campo dos métodos que esta viragem conceptual provocou.

\section{MÉTODOS REVISTOS PARA UMA GEOGRAFIA DO QUE ACONTECE}

A viragem conceptual que as teorias não-representacionais provocaram na Geografia Cultural também configura uma crítica e uma mudança dos métodos de pesquisa. Os métodos de pesquisa passam a apontar para o estudo das formações sociais, preocupando-se menos com estabelecer factos e mais com 
descrever realidades. As reflexões de Latour (2005) são centrais neste aspeto. Segundo Latour (2005), as ciências sociais têm-se focado tradicionalmente em determinar ' matters of fact', definindo a priori o seu campo de estudo (as suas fronteiras, os seus atores, etc.), tentando isolar elementos do mundo social para determinar factos. Para Latour (2005), no entanto, estas matters of fact são apenas uma pequena parte do mundo social, sendo necessário compreender não só o que os torna possível, mas também a sua emergência. Latour (2005) propõe então um foco em matters of concern, isto é, nas formações sociais, no modo como atores (humanos e não humanos) e actantes (objetos, tecnologias, discursos que interferem na ação social) se conjugam na performatividade do dia a dia. É nesta sequência que emerge um novo interesse em metodologias etnográficas e descritivas (Anderson, 2009) mas também que as próprias ideias de método (Law, 2004; Manning, 2015) e campo (McCormack, 2013) são criticadas e repensadas, como já discutimos.

Métodos convencionais tradicionais na Geografia Cultural como a entrevista ou o inquérito, e o processo de "sair para o mundo, fazer um relatório, e depois analisar eventos" têm sido visto como inadequados para "a tarefa de captar as lógicas afetivas e processuais dos espaços-tempos nos quais corpos em movimento são participantes generativos" (McCormack, 2013, p. 118). Daí surge um experimentalismo em termos de método e metodologia que procura meios para captar o dinamismo da vida e teorizar sobre fluxos sensoriais, atmosferas afetivas, assemblages, ritmos e eventos à medida que estes acontecem (Law, 2004; McCormack, 2013; Vannini, 2015a; Manning, 2015).

Alguns métodos tradicionais, como a entrevista, têm sido revistos para se adequarem ao estudo das afectações, da materialidade relacional, e do movimento (Dowling, Lloyd, \& Suchet-Pearson, 2016a). Mas de uma perspetiva geral, é um investimento consistente na etnografia que sobressai, recuperando-se a tradição da observação participante da Antropologia e Sociologia (Anderson, 2009). Deste investimento na etnografia, é particularmente premente abordar duas linhas metodológicas que se têm destacado: os métodos atmosféricos e os métodos móveis. Estes não se podem definir exatamente como um conjunto de técnicas, mas sim como um tipo de aproximação metodológica ao objecto de estudo. Tratamos estes separadamente nas secções seguintes.

\section{Métodos atmosféricos}

Desde os argumentos de Thrift e Dewsbury (2000) até textos mais recentes (Vannini, 2015a), a observação participante tem sido visto como o principal meio para captar a performatividade do dia a dia, pois coloca o investigador diretamente no centro da ação e permite-lhe teorizar a partir dessa posição. Pela importância dos processos sensoriais e afetivos, um corpo significativo de literatura baseia-se em autoetnografias, isto é, no estudo da própria experiência pessoal do investigador em determinada ação social (e.g. Henriques, 2010; Ash, 2012a; 2012b; Simpson, 2013), que deve ser crítico e reflexivo em relação à sua própria posicionalidade devido à extrema subjectividade das observações (Pink, 2009). Esta linha de investigação tem sido conceptualizada como 'métodos atmosféricos', na medida em que usam o corpo do investigador e o seu potencial afetivo para estudar as intensidades das atmosferas dos lugares e as suas causas e consequências (Anderson \& Ash, 2015; McCormack, 2015a).

Métodos atmosféricos têm sido utilizados com objectos de estudo muito diversos incluindo videojogos (Ash, 2012a; 2012b), performances artísticas (Simpson, 2012), ou a experiência de túmulos (Sørensen, 2015). Os métodos baseiam-se na experiência direta dos eventos ou objetos em análise, e na descrição crítica desses eventos, procurando salientar os principais fluxos afetivos em causa que explicam a causalidade dos efeitos das atmosferas nos indivíduos (Anderson \& Ash, 2015). Frequentemente, a análise ancora-se em conceitos da filosofia pós-fenomenológica (Thrift, 2011; Spinney, 2015).

Um aspeto de interesse nestas metodologias é o recurso a tecnologias de registo, nomeadamente a fotografia (Simpson, 2012), o video (Lorimer, 2010; Varotto \& Rosetto, 2016), ou a fonografia (Gallagher 
\& Prior, 2014; Gallagher, 2015), como meio auxiliar de captação dos movimentos e fluxos sensoriais do quotidiano. Estas tecnologias trazem valor para a investigação de duas maneiras. Por um lado, permitem registar a própria participação do investigador na ação social, para uma posterior análise da posição e do papel do investigador na acção social. Simpson (2012; 2013), por exemplo, usou fotografia repetida com lapso temporal para estudar como a sua própria performance de rua afetava os ritmos do espaço público e gerava novas socialidades. Por outro lado, estes registos funcionam como auxílio de memória corporal ao permitirem reviver as afetações emergentes quando são visionados ou ouvidos (Duffy \& Waitt, 2011; Gallagher \& Prior, 2014).

Outro aspeto a salientar no âmbito dos métodos atmosféricos é a questão da intersubjetividade (Simpson, 2015). Isto é, quando o foco recai somente na experiência do investigador, não se aborda toda a diversidade de afetos e reacções em relação aos eventos do mundo. Tendo isso em conta, alguns estudos têm-se ancorado em pesquisa participada, em que o investigador trabalha em proximidade com um conjunto de voluntários que recolhem e discutem informação com o investigador (e.g. Duffy \& Waitt, 2013; MacPherson et al., 2016; Dowling, Lloyd, \& Suchet-Pearson, 2016b).

\section{Métodos móveis}

Com ligação às possibilidades oferecidas por novas tecnologias, tem emergido um conjunto de métodos a que se tem convencionado chamar 'métodos móveis' (Büscher \& Urry, 2009; Merriman, 2014), que procuram criar instrumentos de observação dos indivíduos e das suas práticas no decorrer do seu dia a dia (invariavelmente móvel). Estes enquadram-se na 'etnografia móvel' sugerida por Sheller e Urry (2006) para abordar a vida social num contexto em que a mobilidade de pessoas e bens é uma característica fundamental dos espaços. Destaca-se em primeiro lugar a utilização do diário como método privilegiado para criar um registo das impressões e das práticas dos indivíduos no seu dia a dia. O texto do diário, criado por um participante na investigação, é geralmente utilizado posteriormente para entrevistar os participantes, com o propósito de aprofundar as informações sobre a sua experiência geográfica (Latham, 2003). O uso do diário como método tem sido também complementado com o registo de fotografia, video ou fonografia por participantes, sendo estes dados também geralmente utilizados para elicitar as experiências registadas em conversas ou entrevistas com os participantes, de modo a discutir os seus afetos de um modo mais detalhado (Duffy \& Waitt, 2013; Paiva, 2016a; 2016b).

Em contraste com o método do diário, no qual o participante regista a sua experiência no seu dia a dia sem a presença do investigador, destaca-se também o interesse crescente de investigadores em acompanhar indivíduos no seu dia a dia. Neste âmbito, o acompanhamento faz-se em diferentes intensidades. Num pólo temos as etnografias em movimento, como é o caso de Smith e Hall (2013) que acompanharam patrulhas urbanas que estão disponíveis 24 horas por dia, ou os go-along, em que o investigador acompanha indivíduos no seu dia a dia (Kusenbach, 2003). Noutro pólo, temos pequenas excursões geográficas programadas pelo investigador, como é o caso do soundwalk que tem sido utilizado por geógrafos para explorar a experiência e as práticas situadas através do som (Butler, 2006).

Ainda no âmbito dos 'métodos móveis', é importante referir a emergência de novas tecnologias com bio-sensores capazes de providenciar novos tipos de dados que nos permitem captar processos não-representacionais no quotidiano. Embora este ainda seja um campo incipiente, verifica-se um interesse crescente na Geografia Cultural em trabalhar com bio-sensores móveis que permitem obter dados sobre as respostas fisiológicas dos indivíduos ao ambiente geográfico e à vida social. Entre estas tecnologias destacam-se o eye-tracking, que permite registar o foco da visão dos indivíduos (Beljaars, 2015); sensores de batimentos cardíacos (Ferreira \& Salvador, 2012); o eletroencefalograma (Aspinall, Mavros, Coyne, \& Roe, 2013), que nos oferece informações sobre a atividade cerebral através da leitura de estímulos elétricos no cérebro; e os sensores de resposta galvânica da pele que medem as reacções emocio- 
nais através do registo da condutância elétrica da pele (Spinney, 2015). O tamanho destes aparelhos tem diminuído nos últimos anos, permitindo o registo móvel com uma interferência mais reduzida no dia a dia dos indivíduos, havendo também versões com registo de dados GPS. A proliferação de produtos low-cost nesta categoria tem tornado o seu uso mais acessível (Spinney, 2015).

\section{CONCLUSÃO}

Existem duas considerações que importa fazer sobre acerca do impacto das teorias não-representacionais na Geografia Cultural. Em primeiro lugar, esta perspetiva teórica procura trazer o investigador para o centro da ação social, para possibilitar uma compreensão na prática dos fenómenos geográficos. Este esbater da distinção entre teoria e prática nas teorias não-representacionais provocou um maior experimentalismo em termos de metodologias e métodos, que por sua vez resulta num maior leque de métodos e técnicas utilizadas por geógrafos. Deste modo, as teorias não-representacionais permitem entender aspetos antes negligenciados na experiência espacial, tendo obtido sucesso particularmente no estudo das práticas espaciais e a sua experiência.

Em segundo lugar, a abrangência das teorias não-representacionais e a desestabilização conceptual e metódica que provocam deu à Geografia Cultural mais instrumentos conceptuais e metodológicos para abordar os aspectos qualitativos de determinados fenómenos. Hoje, através da lente das teorias não-representacionais, abordam-se temas tão diversos como as geografias dos transportes e mobilidades (Bissel, 2010; Vannini, 2012; Stewart, 2014), interfaces tecnológicos (Ash, 2015), o papel dos sentidos na experiência geográfica (Middleton, 2010), doenças mentais (Blackman, 2010; Beljaars, 2015), adições (Bøhling, 2015), processos legais como adopções (Posocco, 2011); tortura de prisioneiros políticos (Anderson, 2014), preparações governamentais para emergências futuras (Anderson, 2010), ou fenómenos económicos como a inflação (McCormack, 2015b).

Apesar disto, existem algumas críticas que têm sido apontadas à aplicação das teorias não-representacionais na geografia. Uma delas é o facto de nem sempre os investigadores conseguirem teorizar a partir da experiência e dos eventos do mundo, ou pelo menos demonstrar como a sua teoria parte daí, sendo antes teorias oriundas da filosofia pós-estruturalista e da pós-fenomenologia que emergem em primeiro plano nos artigos e livros publicados (Cresswell, 2012a). Daí resultam estudos que pouco acrescentam à compreensão dos eventos e das experiências do dia a dia. Outra crítica comum é o facto de por vezes os estudos no âmbito das teorias não-representacionais terem um tom universalista e não tomarem em conta distinções de género, etnicidade, ou classe social que podem estar na origem ou afetar os fenómenos que estudam (Tolia-Kelly, 2006; Lorimer, 2015). Por último, e relacionado com o argumento anterior, o foco na especificidade das relações entre corpos e espaço impede frequentemente generalizações acerca dos processos que estuda.

O desenvolvimento das teorias não-representacionais tem o seu core no Reino Unido (Anderson \& Harrison, 2010), e em particular na Universidade de Bristol (Cresswell, 2012b; Lorimer, 2015), mas a sua influência estende-se por todo o mundo anglófono - Estados Unidos da América (Stewart, 2011; 2014; Campbell, 2016), Canadá (Vannini, 2012; 2015a), Austrália (Bissell, 2010; 2015) - bem como outros países da Europa, (Bille, 2015; Pløger, 2015; Sørensen, 2015; Varotto \& Rossetto, 2016).

Em Portugal, podem-se identificar uma série de trabalhos que recorrem a estas teorias e às suas metodologias. Um dos primeiros trabalhos substanciais surge com a publicação da coletânea Geografias do Corpo (Azevedo, Pimenta, \& Sarmento, 2009), onde vários estudos abordam as implicações geográficas do corpo ligadas às temáticas do poder, das representações, e das práticas. Desde então, outros trabalhos têm sido publicados. Carmo (2011) mobilizou teorias não-representacionais no estudo da performatividade de murais políticos na paisagem urbana. Ferreira e Salvador (2012; 2015; ver também 
Ferreira, 2013; Silva \& Ferreira, 2014) abordaram as geografias lésbicas em Portugal com recurso a cartografias pós-representacionais. Também Azevedo, Martins, Carneiro, Acosta, Pereira, e Pinto (2014) discutem o uso de cartografias pós-representacionais para o estudo da experiência da paisagem. Silva et al. (2015) estudam o papel do corpo e dos sentidos na educação. Por último, os ritmos urbanos têm também sido objecto de análise por geógrafos portugueses (Paiva; 2016a; 2016b; Sarmento, 2017; Paiva, Cachinho, Barata Salgueiro \& Amílcar, 2017). Quase dez anos depois da publicação de Geografias do Corpo, poderá questionar-se se não será oportuna a organização de um novo volume que reúna os diversos estudos publicados no campo do não- ou mais-que-representacional em Portugal, hoje esparsos em diversas publicações.

Apesar de se terem tornado uma perspetiva relevante na Geografia Cultural, as teorias não-representacionais não apagaram outras perspetivas - a Geografia Cultural continua a ter como característica fundamental a pluralidade de visões -, embora a sua influência seja incontornável na investigação qualitativa nesta ciência (Cresswell, 2012b; Lorimer, 2015).

\section{REFERÊNCIAS BIBLIOGRÁFICAS}

Anderson, B. (2014). Encountering Affect. Capacities, Apparatuses, Conditions. Surrey: Ashgate.

Anderson, B. (2010). Preemption, precaution, preparedness: Anticipatory action and future geographies. Progress in Human Geography, 34(6), 777-798.

Anderson, B., \& Ash, J. (2015). Atmospheric methods. In P. Vannini (Ed.), Non-Representational Methodologies: Re-Envisioning Research (pp. 34-51). London: Routledge.

Anderson, B., \& Harrison, P. (Ed.) (2010). Taking-place: non-representational theories and geography. Surrey: Ashgate.

Anderson, J. (2009). Understanding cultural geography: places and traces. New York: Routledge.

Ash, J. (2015). The Interface Envelope: Gaming, Technology, Power. London: Bloomsbury.

Ash, J. (2012a). Technology, technicity, and emerging practices of temporal sensitivity in videogames. Environment and Planning A, 44, 187-203.

Ash, J. (2012b). Attention, videogames and the retentional economies of affective amplification. Theory, Culture \& Society, 29(6), 3-26.

Aspinall, P., Mavros, P., Coyne, R., \& Roe, J. (2013). The urban brain: analysing outdoor physical activity with mobile EEG. British Journal of Sports Medicine. Retrieved from http://bjsm.bmj.com/content/early/2013/03/05/bjsports-2012-091877.full

Azevedo, A. F., Pimenta, J. R., \& Sarmento, J. (2009). Geografias do corpo. Ensaios de Geografia Cultural [Body Geographies. Essays in Cultural Geography]. Porto: Figueirinhas.
Azevedo, A. F., Martins, R., Carneiro, P., Acosta, R., Pereira, P., \& Pinto, M. (2014). Caixa Infernal: cartografias entre o visual e para além do representacional ou dispositivo para a organização da experiência de paisagem [Infernal Box: cartographies between the visual and the morethan-representational or the dispositif for organizing landscape experience]. In A. Vieira, \& R. P. Julião. (Eds.), 'A Jangada de Pedra'. Geografias Ibero-Afro-Americanas. Atas do XIV Colóquio Ibérico de Geografia (386-392). Guimarães: Associação Portuguesa de Geógrafos e Departamento de Geografia da Universidade do Minho.

Beljaars, D. (2015, September). Eye-tracking: Retracing visual perception in the everyday environments of people with Tourette syndrome. RGS-IBG Annual International Conference 2015, Exeter.

Bille, M. (2015). Lighting up cosy atmospheres in Denmark. Emotion, Space and Society, 15, 56-63.

Bissell, D. (2015). Virtual infrastructure of habit: the changing intensities of habit through gracefulness, restlessness and clumsiness. Cultural Geographies, 22(1), 127-146.

Bissel, D. (2010). Passenger mobilities: affective atmospheres and the sociality of public transport. Environment and Planning D: Society and Space, 28, 270-289.

Blackman, L. (2010). Embodying Affect: Voice-hearing, Telepathy, Suggestion and Modelling the Nonconscious. Body \& Society, 16(1), 163-192. 
Bøhling, F. (2015). Alcoholic assemblages: Exploring fluid subjects in the night-time economy. Geoforum, 58, 132-142.

Brennan, T. (2004). The Transmission of Affect. New York: Cornell University.

Büscher, M., \& Urry, J. (2009). Mobile methods and the empirical. European Journal of Social Theory, 12(1), 99-116.

Butler, T. (2006). A walk of art: the potential of the sound walk as practice in cultural geography. Social \& Cultural Geography, 7(6), 889-908.

Campbell, N. (2016). Affective Critical Regionality. London: Rowman \& Littlefield.

Carmo, A. (2011). Revolutionary landscapes: the PCTP/ MRPP mural paintings in the Lisbon Metropolitan Area. Finisterra - Revista Portuguesa de Geografia, XLVI(92), 25-41.

Crang, M., \& Cook, I. (2007). Doing ethnographies. London: Sage.

Clough, P. (2007). The Affective Turn: Theorizing the Social. Durham: Duke University Press.

Cresswell, T. (2012a). Geographical Thought: A Critical Introduction. London: Wiley.

Cresswell, T. (2012b). Nonrepresentational Theory and Me: Notes of an Interested Sceptic. Environment and Planning D: Society and Space, 30(1), 96-105.

Dowling, R., Lloyd, K., \& Suchet-Pearson, S. (2016a). Qualitative methods I: Enriching the interview. Progress in Human Geography, 40(5), 679-686.

Dowling, R., Lloyd, K., \& Suchet-Pearson, S. (2016b). Qualitative methods II: 'More-than-human' methodologies and/in praxis. Progress in Human Geography, Online First, doi: 10.1177/0309132516664439

Duffy, M., \& Waitt, G. (2011). Sound diaries: a method for listening to place. Aether. The Journal of Media Geography, VII, 119-136.

Duffy, M., \& Waitt, G. (2013). Home sounds: experiential practices and performativities of hearing and listening. Social \& Cultural Geography, 14(4), 466-481.

Ferreira, E. (2013). Reconceptualising public spaces of (in)equality: sensing and creating layers of visibility. (PhD Thesis). Universidade Nova de Lisboa, Lisboa. Retrieved from https://run.unl.pt/handle/10362/11913

Ferreira, E., \& Salvador, R. (2015). Lesbian collaborative web mapping: disrupting heteronormativity in Portugal. Gender, Place \& Culture: A Journal of Feminist Geography, 22(7), 954-970.
Ferreira, E., \& Salvador, R. (2012). Sensing the Landscape: Collaborative Emotion Mapping in Urban Spaces. In XIII Colóquio Ibérico de Geografia (587-597), Santiago de Compostela.

Gallagher, M. (2015). Field recording and the sounding of spaces. Environment and Planning D: Society and Space, 33(3), 560-576.

Gallagher, M., \& Prior, J. (2014). Sonic geographies: Exploring phonographic methods. Progress in Human Geography, 38(2), 267-284.

Greenhough, B. (2010) Vitalist Geographies: Life and the More-Than-Human. In B. Anderson, \& P. Harrison (Eds.), Taking-place: non-representational theories and geography (pp. 37-54). Surrey: Ashgate.

Haraway, D. (1988). Situated knowledges: the science question in feminism and the privilege of partial perspective. Feminist Studies, 14(3), 575-599.

Henriques, J. (2010). The vibrations of affect and their propagation on a night out on Kingston's dancehall scene. Body \& Society, 16(1), 57-89.

Kusenbach, M. (2003). Street phenomenology. The goalong as ethnographic research tool. Ethnography, 4(3), 455-485.

Latham, A. (2003). Research, performance, and doing human geography: some reflections on the diary-photograph, diary-interview method. Environment and Planning A, 35(11), 1993-2017.

Latour, B. (2005). Reassembling the Social. An Introduction to Actor-Network-Theory. Oxford: Oxford University Press.

Law, J. (2004). After Method. Mess in social science research. Oxon: Routledge.

Lorimer, H. (2015). Afterword: Non-representational theory and me too. In P. Vannini (Ed.), Non-Representational Methodologies: Re-Envisioning Research (pp. 177-188). London: Routledge.

Lorimer, J. (2010). Moving image methodologies for more-than-human geographies. Cultural geographies, 17(2), 237-258.

MacPherson, H., Fox, A., Street, S., Cull, J., Jenner, T. Lake, D.,... Hart, S. (2016). Listening space: lessons from artists with and without learning disabilities. Environment and Planning D: Society and Space, 34(2), 371-389.

Manning, E. (2015). Against method. In P. Vannini (Ed.), Non-Representational Methodologies: ReEnvisioning Research (pp. 52-71). London: Routledge. 
McCormack, D. (2015a). Devices for doing atmospheric things. In P. Vannini (Ed.), Non-Representational Methodologies: Re-Envisioning Research (pp. 89-111). London: Routledge.

McCormack, D. (2015b). Governing Inflation: Price and Atmospheres of Emergency. Theory, Culture \& Society, 32(2), 131-154.

McCormack, D. (2013). Refrains for Moving Bodies: Experience and Experiment in Affective Spaces. Durham: Duke University Press.

Merriman, P. (2014). Rethinking Mobile Methods. Mobilities, 9(2), 167-187.

Middleton, J. (2010). Sense and the city: exploring the embodied geographies of urban walking, Social \& Cultural Geography, 11(6), 575-596.

Paiva, D. (2017). Teorias não-representacionais na Geografia I: conceitos para uma geografia do que acontece [Non-representational theories in geography I: concepts for a geography of what happens] Finisterra - Revista Portuguesa de Geografia, LII(106), 159-168.

Paiva, D. (2016a). Collapsed rhythms: the impact of urban change in the everyday life of elders. Space and Culture, 19(4), 345-360.

Paiva, D. (2016b). Assessing sonic affects in everyday life: looking for metacognition and metaemotion. Qualitative Research Journal, 16(S1), 80-91.

Paiva, D., Cachinho, H., Barata Salgueiro, T., \& Amílcar, A. (2017). A criação de geoetnografias como metodologia para o estudo dos ritmos urbanos. Uma aplicação no Chiado, Lisboa [The creation of geoethnographies as a research methodology for urban rhythms. An application on Chiado, Lisbon]. Scripta Nova - Revista Eletrónica de Geografía y Ciencias Sociales, XXI(569), 1-29.

Pink, S. (2009). Doing Sensory Ethnography. London: Sage.

Posocco, S. (2011). Expedientes: Fissured legality and affective states in the transnational adoption archives in Guatemala. Journal of Law, Culture and Humanities, 7, 434-456.

Posteraro, T. S. (2014). Organismic Spatiality: Toward a Metaphysic of Composition. Environment and Planning D: Society and Space, 32(4), 739-752.

Sarmento, J. (2017). Tourists' walking rhythms: 'doing' the Tunis Medina, Tunisia. Social \& Cultural Geography, 18(3), 295-314.

Silva, M. J., \& Ferreira, E. (2014). Abordagens corporizadas, com recurso às TIC, na investigação em geografias de género e da sexualidade [Bodily approaches using ICT in research in geographies of gender and sexualities]. In A. Vieira, \& R. P. Julião (Eds.), 'A Jangada de Pedra'. Geografias Ibero-Afro-Americanas. Atas do XIV Colóquio Ibérico de Geografia (pp. 2510-2515). Guimarães: Associação Portuguesa de Geógrafos e Departamento de Geografia da Universidade do Minho.

Silva, M. J., Ferreira, E., Andrade, V., Nunes, O., \& Carvalho, M. (2015). Embodied education: Senses, emotions, and technology. In 2015 International Symposium on Computers in Education (SIIE). Setúbal: School of Education of the Polytechnic Institute of Setúbal.

Simpson, P. (2015). What remains of the intersubjective? On the presencing of self and other. Emotion, Space and Society, 14, 65-73.

Simpson, P. (2013). Ecologies of Experience: Materiality, Sociality, and the Embodied Experience of (Street) Performing. Environment and Planning A, 45(1), 180-196.

Simpson, P. (2012). Apprehending everyday rhythms: rhythmanalysis, time-lapse photography, and the space-times of street performance. cultural geographies, 19(4), 423-445.

Smith, R. J., \& Hall, T. (2013). No time out: mobility, rhythmicity and urban patrol in the twenty-four hour city. The Sociological Review, 61(S1), 89-108.

Sørensen, T. M. (2015). More than a feeling: Towards an Archaeology of Atmosphere. Emotion, Space and Society, 15, 64-73.

Spinney, J. (2015). Close encounters? Mobile methods, (post)phenomenology and affect. cultural geographies, 22(2), 231-246.

Stewart, K. (2014). Road registers. cultural geographies, 21(4), 549-563.

Stewart, K. (2011). Atmospheric Attunements. Environment and Planning D: Society and Space, 29(3), 445-453.

Thrift, N. (2011). Lifeworld inc. Environment and Planning D: Society and Space, 29(1), 5-26.

Thrift, N. (2008). Non-representational Theory. Space, Politics, Affect. Oxon: Routledge.

Thrift, N., \& Dewsbury, J. (2000). Dead Geographies And How to Make Them Live. Environment and Planning D: Society and Space, 18(4), 411-432.

Tolia-Kelly, D. (2006). Affect - an ethnocentric encounter? Exploring the 'universalist' imperative or emotional $\backslash$ affective geographies. Area, 38, 213-217.

Vannini, P. (2015a). Non-Representational Methodologies: Re-Envisioning Research. London: Routledge. 
Vannini, P. (2015b). Non-representational ethnography: new ways of animating lifeworlds. Cultural Geographies, 22(2), 317-327.

Vannini, P. (2012). In Time, Out of Time: Rhythmanalyzing Ferry Mobilities. Time \& Society, 21(2), 241-269.
Varotto, M., \& Rossetto, T. (2016). Geographic Film as Public Research: Re-Visualising/Vitalising a Terraced Landscape in the Italian Alps (Piccola terra/Small land, 2012). Social and Cultural Geography, 17(8), 1140-1164.

i Paiva, D. (2017). Teorias não-representacionais na Geografia I: conceitos para uma geografia do que acontece Finisterra Revista Portuguesa de Geografia, LII(106), 159-168.

ii $\mathrm{O}$ foco na Geografia Cultural justifica-se por ser neste campo que as teorias não-representacionais tiveram maior impacto. Em acréscimo, os estudos não- ou mais-que-representacionais noutras disciplinas (e.g. Geografia Política, Geografia Económica, ou Geografia Urbana) têm geralmente uma perspetiva sobre os fenómenos enquanto cultura, ou 'mais-que-cultura'.

iii Todas as citações neste texto a partir de originais em língua inglesa são tradução minha. 